\title{
Clostridium colinum sp. nov., nom. rev., the Causative Agent of Ulcerative Enteritis (Quail Disease) in Quail, Chickens, and Pheasants
}

\author{
HERMAN A. BERKHOFF \\ Department of Microbiology, Pathology, and Parasitology, School of Veterinary Medicine, North Carolina State \\ University, Raleigh, North Carolina 27606
}

\begin{abstract}
The causative agent of ulcerative enteritis (quail disease) is described, and the name Clostridium colinum is revived for this organism. The type strain is strain 72042 (= ATCC 27770 ).
\end{abstract}

Clostridium colinum, the causative agent of ulcerative enteritis (quail disease), was named and described in 1974 by Berkhoff et al. (3). However, this name did not appear on the Approved Lists of Bacterial Names (16), and the name currently has no taxonomic standing. In this paper a description of the species is provided, and the name Clostridium colinum is revived for the same strains and species described originally. The type strain of Clostridium colinum is strain 72042 (= ATCC 27770).

\section{MATERIALS AND METHODS}

Isolation of strains. The procedures used for bacterial isolation were those described by Berkhoff et al. (3).

Cultures were obtained directly from the livers of naturally infected birds that had died of ulcerative enteritis (14). A total of 23 strains were obtained from White Leghorn chickens (a commercial laying strain); 1 strain was obtained from an outbreak of the disease in pheasants (Phasianus colchicus), and three cultures were isolated from bobwhite quail (Colinus virginianus). The GasPak system (BBL Mi- crobiology Systems, Cockeysville, Md.) (5) was the anaerobic culture technique used. The basal agar used was supplemented brain heart infusion agar (8) modified by adding the following reducing agents: palladium chloride (0.33 g/liter) (7) and dithiothreitol $(0.1$ g/liter) $(6,7,13)$. Anaerobic blood agar plates (BAP) were prepared by adding $7 \%$ citrated horse blood to the basal medium. Anaerobic egg yolk agar was prepared by using the formula of Holdeman et al. (8). The same reducing agents used for brain heart infusion agar were added to this medium.

Culture methods for characterization. The roll tube system (9) with prereduced anaerobically sterilized media (8) was used together with a Virginia Polytechnic Institute and State University anaerobic culture system (Bellco Glass, Inc., Vineland, N.J.) for the bacteriological characterization studies.

Media. The culture media used with the roll tube system, which were prepared as described in the Anaerobe Laboratory Manual (8), were BAP and modified McClung-Toabe egg yolk agar. The BAP and egg yolk agar plates were streaked as soon as the agar solidified, and then they were incubated immediately in an anaerobic jar. Therefore, these

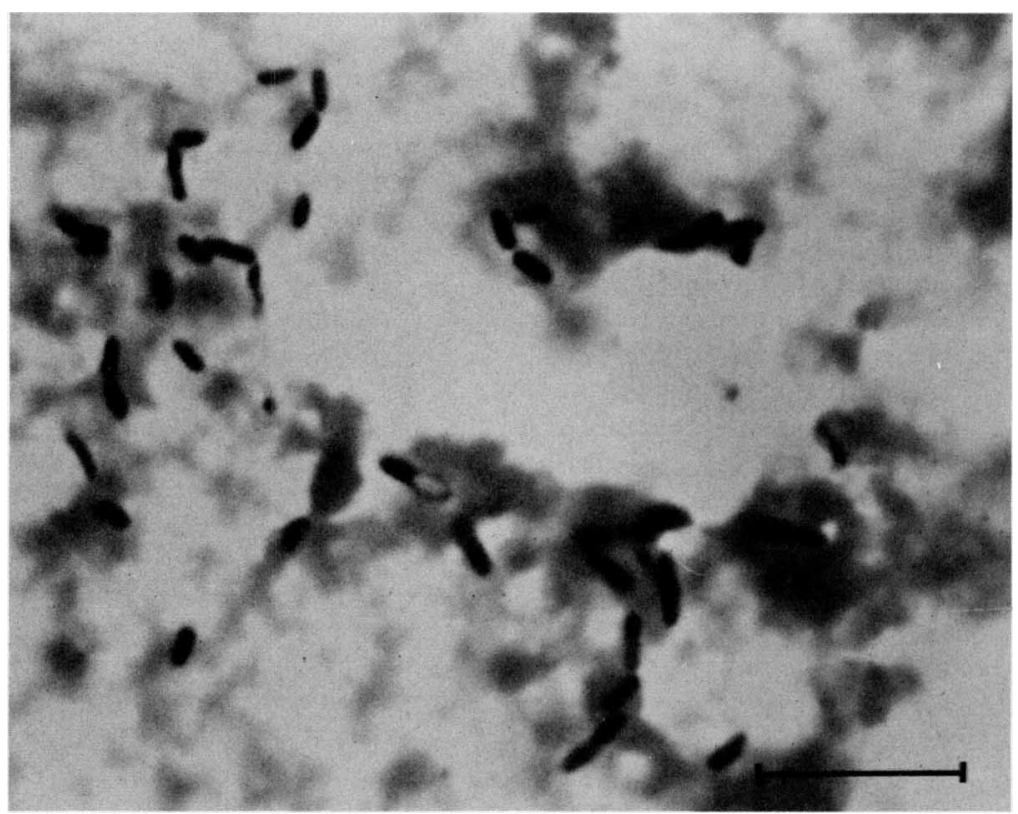

FIG. 1. Vegetative and sporulated cells of Clostridium colinum strain ATCC $27770^{\mathrm{T}}(\mathrm{T}=$ type strain). Gram stain of a 24-h anaerobic BAP culture. Bar $=10 \mu \mathrm{m}$. 


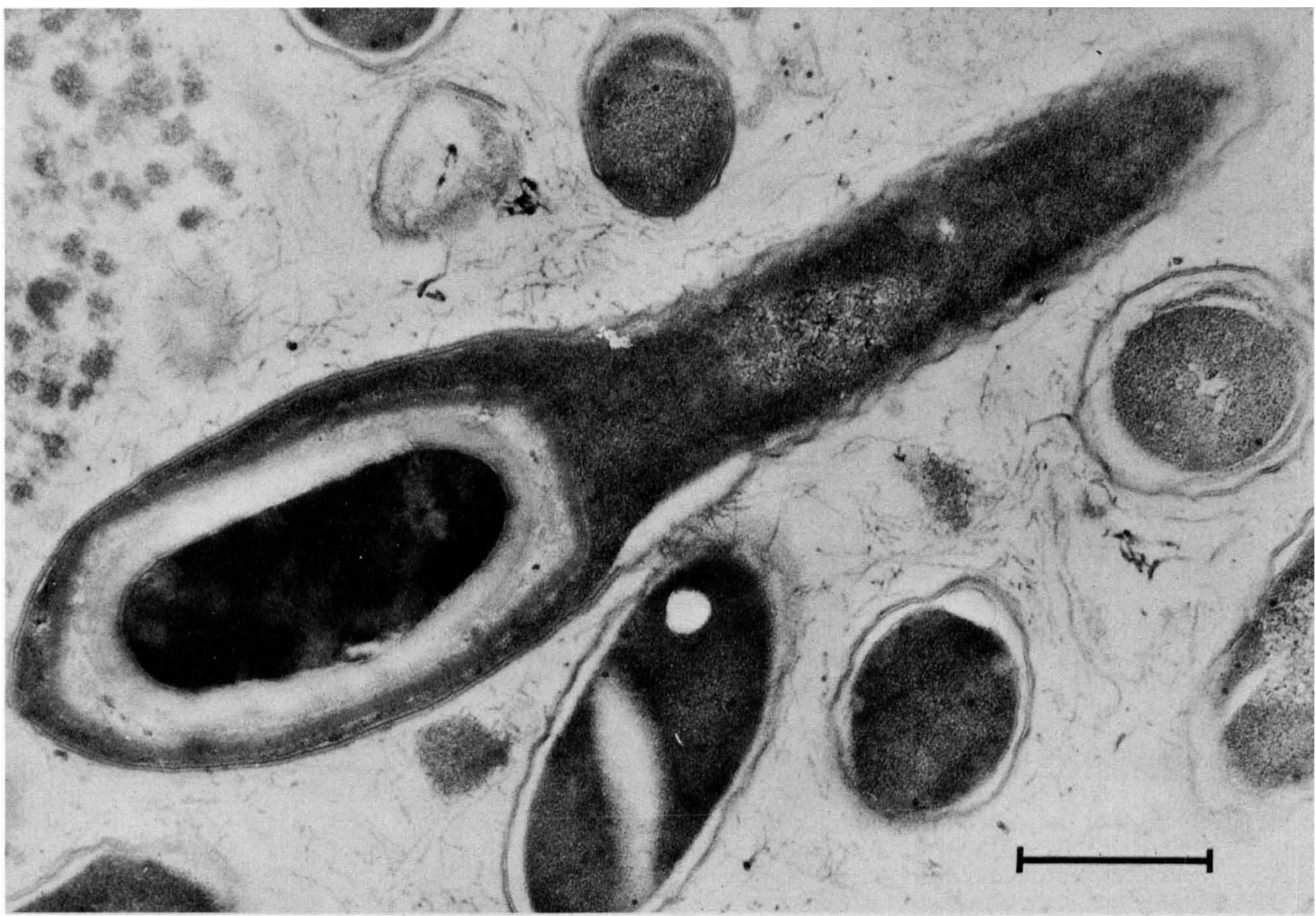

FIG. 2. Electron micrograph of Clostridium colinum showing typical bacterial spore structures and vegetative cells. Bar $=1 \mu \mathrm{m}$.

plate media were not truly prereduced; if they were not used within $4 \mathrm{~h}$ of preparation, they were stored in anaerobic jars. Peptone-yeast extract broth was the basal medium used for the characterization studies with the roll tube system. Carbohydrates were added at final concentrations of either 0.5 $\mathrm{g} / \mathrm{ml}$ or $1.0 \mathrm{~g} / 100 \mathrm{ml}$. Chopped meat-carbohydrate medium (CMC medium), gelatin medium, $\mathrm{H}_{2} \mathrm{~S}$ medium, and indolenitrate medium (BBL) were also used. Indole-nitrate medium was not prepared prereduced. Indole production was also detected in CMC medium. The Virginia Polytechnic Institute and State University anaerobic culture system was used together with the prereduced anaerobically sterilized culture method. Special anaerobic culture tubes that were tightly sealed with rubber stoppers were employed.

Reagents for tests. Ehrlich reagent was used to test for indole production. To detect the reduction of nitrate, the reagents used were sulfanilic acid and dimethyl-alphanaphthylamine. Ferric ammonium citrate was used to test for esculin hydrolysis.

pH. $\mathrm{pH}$ was measured directly in the culture tubes with a long, thin combination electrode (catalog no. 39013; Beckman Instruments, Inc., Fullerton, Calif.) (8).

Analysis of fermentation products. A Beckman model GC-2A gas chromatograph was used to analyze the fermentation products. The analysis was done by using $\mathrm{CMC}$ medium as described by Holdeman et al. (8).

Spores. To test the heat resistance of spores, peptoneyeast extract broth medium containing $1.0 \%$ starch was inoculated and heated in a water bath at $80^{\circ} \mathrm{C}$ for $10 \mathrm{~min}(8$, 17). To test resistance of spores to octanol and chloroform, 5.0-ml cultures containing spores were shaken with 1 drop of octanol or 1 drop of chloroform for $5 \mathrm{~min}$ (1).

Electron microscopy. A drop of an actively growing broth culture or of a BAP colony suspended in saline was placed directly onto a copper grid coated with carbon. The preparation was stained with $1 \%$ phosphotungstic acid. Flagella were also examined in unstained preparations that were shadowed with platinum. All sections were examined, and electron photomicrographs were taken with an RCA model EMU-3G electron microscope.

Determination of deoxyribonucleic acid base composition (guanine-plus-cytosine content). The average guanine-pluscytosine content of each deoxyribonucleic acid was measured by the thermal denaturation method (10) and was calculated by using the equation of Marmur and Doty (11).

Growth measurements. A normal growth curve for the anaerobe was established by using the following two methods: optical density determined with a Spectronic 20 photocolorimeter and viable counts. The number of viable bacterial cells was determined by counting the colonies formed on the surface of BAP; 10 -fold dilutions of cultures were made in skim milk (Difco Laboratories, Detroit, Mich.), and then they were inoculated onto BAP by dropping $0.05-\mathrm{ml}$ portions with a serological pipette $(0.2 \mathrm{ml}$ graduated in $0.01-\mathrm{ml}$ increments) onto the medium. Four replicates were used for each dilution, and titers were expressed as colony-forming units per milliliter of inoculum.

Toxigenicity. Both actively growing $\mathrm{CMC}$ medium cultures ( 6 to $10 \mathrm{~h}$ ) and old CMC medium cultures ( 72 to $96 \mathrm{~h}$ ) were tested (17). Either the cultures were centrifuged at $23,000 \times$ 


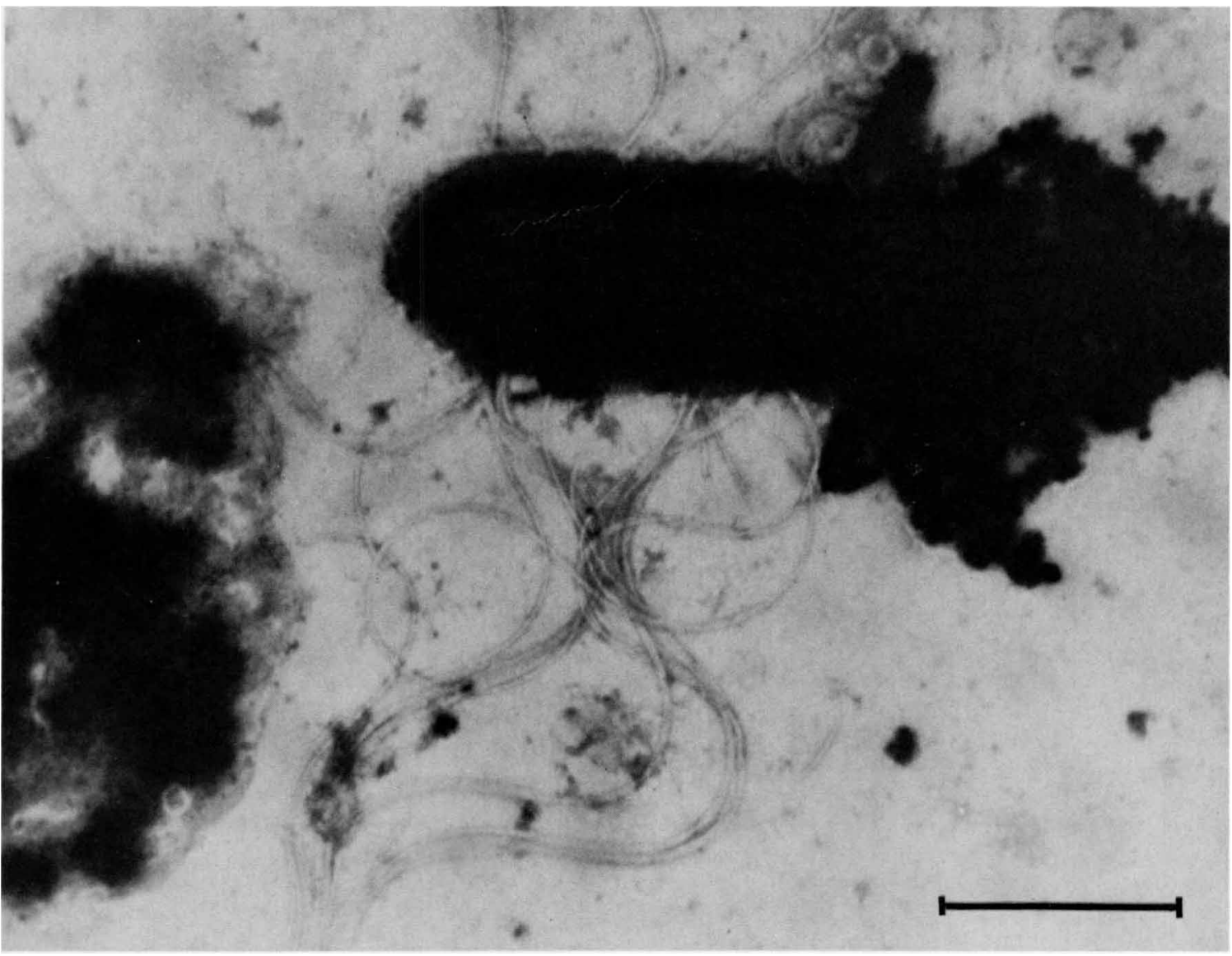

FIG. 3. Electron micrograph of Clostridium colinum showing its peritrichous flagella. Bar $=1 \mu \mathrm{m}$.

$g$ for $10 \mathrm{~min}$ in a refrigerated centrifuge and the supernatant fluid was carefully collected, or they were filtered through a 0.45- $\mu \mathrm{m}$ grid membrane filter unit (Nalgene Labware Div., Nalge/Sybron Corp., Rochester, N.Y.). The supernatants obtained were divided into two portions; one portion remained untreated, and the other was treated with $0.1 \%$ trypsin and incubated at $37^{\circ} \mathrm{C}$ for $30 \mathrm{~min}$ before being intravenously inoculated into mice (approximately $22 \mathrm{~g}$ each) (17).

Animal assay for pathogenicity. The following methods were used to reproduce the disease in experimental animals (3): 5- to 8-week-old quail (Colinus virginianus) were inoculated orally with a known number of viable organisms (12) (15\% skim milk [Difco] was added to all inocula as a protective substance [2]); for pathogenicity tests in guinea pigs, $0.3-\mathrm{ml}$ portions of actively growing cultures mixed with equal parts of $10 \%$ calcium chloride were inoculated intramuscularly into the hind legs (17).

\section{RESULTS AND DISCUSSION}

The bacterial isolates obtained from the livers of naturally infected birds that had died of ulcerative enteritis (14) appeared to be a distinct species that belongs in Clostridium group III, as described by Smith and Hobbs (18). A description of this species is given below.

Clostridium colinum sp. nov. (co. li' num. L. adj. colinum from the scientific name of bobwhite quail, Colinus virginianus, the avian species that is most susceptible to this anaerobe) cells are anaerobic, gram-positive straight rods that are 3 to $4 \mu \mathrm{m}$ long by 0.6 to $1 \mu \mathrm{m}$ wide. The rods appear singly or in pairs; chains are rarely seen. Spores are present in cultures on BAP, but they are few and hard to find; the spores are elliptical and subterminal. The sporing rods are at least twice as thick and long as the vegetative forms (Fig. 1 and 2). The spores resist a temperature of $80^{\circ} \mathrm{C}$ for $10 \mathrm{~min}$, as

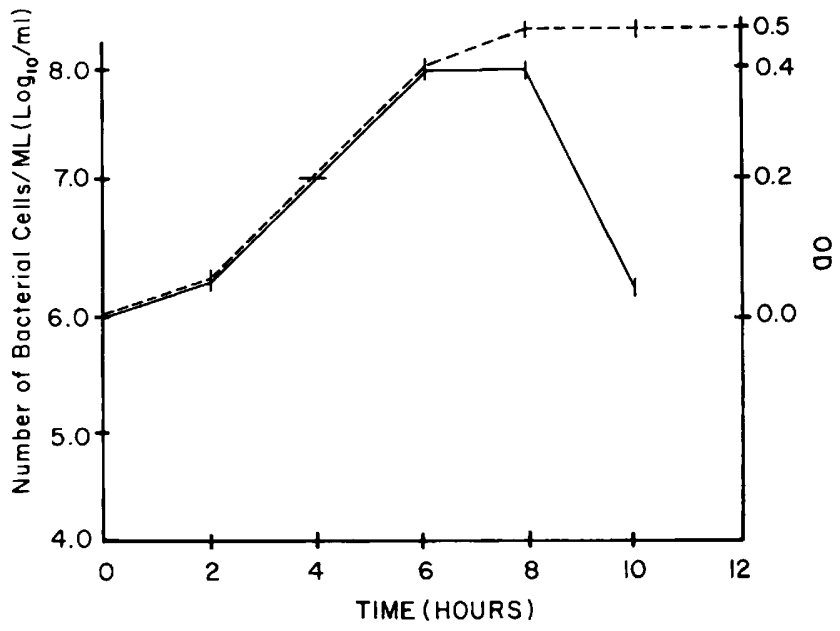

FIG. 4. Normal growth curve of Clostridium colinum in CMC medium. Solid line, viable counts; dashed line, optical density (OD). 


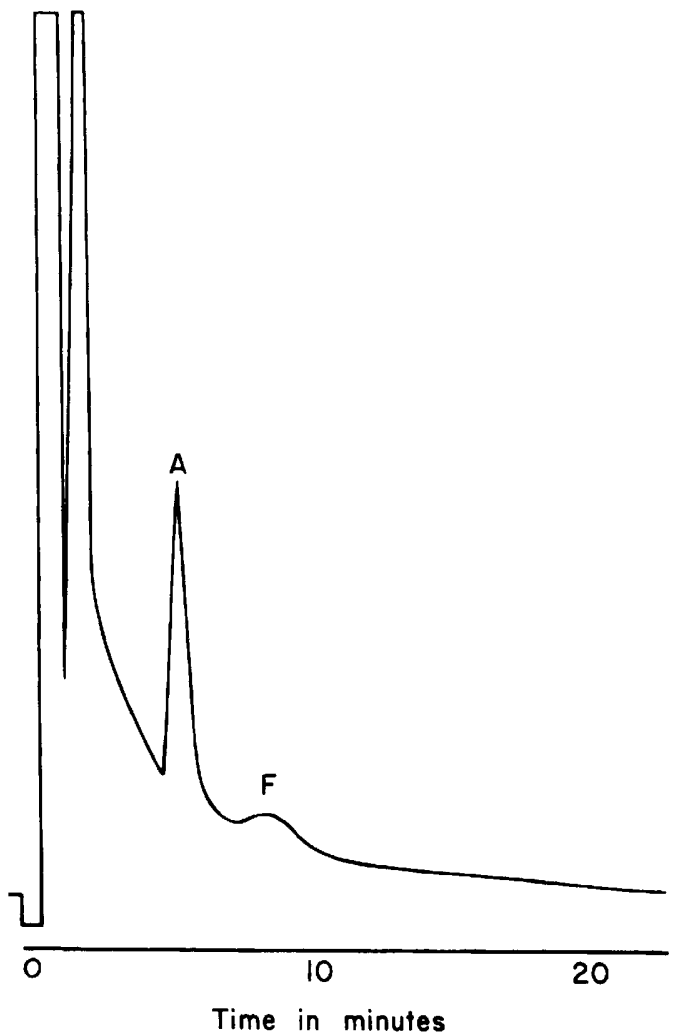

FIG. 5. Typical chromatogram of volatile fatty acids produced by Clostridium colinum. A, Acetic acid; F, formic acid.

well as octanol and chloroform. In broth cultures, spores are also rarely formed. The cells are motile by means of peritrichous flagella (Fig. 3), and they have a cell wall that is typical of the cell walls of gram-positive species.

On solid medium, colonies appear after 1 to 2 days of incubation at $37^{\circ} \mathrm{C}$. Surface colonies on BAP are small, round, semitranslucent, convex, and approximately 1 to 2 $\mathrm{mm}$ in diameter and have filamentous margins. The optimum growth temperature is 35 to $42^{\circ} \mathrm{C}$. Growth in broth media can be detected as early as 12 to $16 \mathrm{~h}$ postinoculation. When a culture is growing actively in broth, it produces gas. Gas production continues for no more than 6 to $8 \mathrm{~h}$. After gas production has stopped, the growth settles in the bottom of the tube. Figure 4 shows a normal growth curve of Clostridium colinum in CMC medium.

The following carbohydrates are fermented: glucose, mannose, raffinose, sucrose, and trehalose. Fructose and maltose are weakly fermented. Mannitol is fermented only by some strains. The type strain does ferment mannitol.

The following carbohydrates are not fermented: arabinose, cellobiose, erythritol, glycogen, inositol, lactose, melezitose, melibiose, rhamnose, sorbitol, and xylose.

Esculin is hydrolyzed; starch hydrolysis is usually negative. Only two strains have been found to cause starch hydrolysis. The type strain does not hydrolyze starch. Nitrite and indole are not produced. Milk is unchanged, and casein is not digested. Good growth occurs in CMC broth. Pyruvate and lactate are not utilized. Gelatin is not liquefied. Catalase, urease, lipase, and lecithinase are not produced.

The fermentation products of this organism are acetic acid and formic acid (Fig. 5).

Morphologically, and to some extent biochemically, Clostridium colinum most closely resembles Clostridium difficile,
Clostridium barati, Clostridium carnis, Clostridium fallax, and Clostridium sticklandii $((8,15,17,18)$. However, all of these species produce butyric acid and would not normally be confused with Clostridium colinum (8). Two other previously described species that are similar to Clostridium colinum are Clostridium clostridiforme and Clostridium oroticum. However, in addition to morphological differences, Clostridium oroticum ferments rhamnose but not trehalose, and both Clostridium clostridiiforme and Clostridium oroticum ferment xylose, unlike Clostridium colinum (8). Clostridium colinum has been isolated only from necrotic liver lesions of quail, pheasants, and chickens dying with lesions typical of ulcerative enteritis (quail disease). Animal assays for pathogenicity have shown that at least $10^{6}$ viable bacterial cells administered orally are required to experimentally reproduce ulcerative enteritis in quail. The bacterium is invariably reisolated from the livers of quail that die from experimentally induced ulcerative enteritis (2). Cultures supplemented with $10 \%$ calcium chloride do not produce any pathogenic effects when they are inoculated into the hind legs of guinea pigs. Cell-free culture filtrates are not toxic for mice. Because infections recur year after year on the same premises, it is assumed that Clostridium colinum survives in the soil of infected premises (14).

The mean guanine-plus-cytosine content of the deoxyribonucleic acid is $43 \mathrm{~mol} \%$. The type strain is strain 72042 (= ATCC 27770).

\section{LITERATURE CITED}

1. Balassa, G. 1966. Synthese et fonction des ARN messagers au cours de la sporulation de Bacillus subtilis. Ann. Inst. Pasteur Paris 110:175-191.

2. Berkhoff, H. A., and S. G. Campbell. 1974. Etiology and pathogenesis of ulcerative enteritis (quail disease). The experimental disease. Avian Dis. 18:205-212.

3. Berkhoff, H. A., S. G. Campbell, and H. B. Naylor. 1974. Etiology and pathogenesis of ulcerative enteritis (quail disease). Isolation of the causative anaerobe. Avian Dis. 18:186-194.

4. Berkhoff, H. A., S. G. Campbell, H. B. Naylor, and L.DS. Smith. 1974. Etiology and pathogenesis of ulcerative enteritis (quail disease). Characterization of the causative anaerobe. Avian Dis. 18:195-204.

5. Brewer, J. H., and D. L. Allgeier. 1966. Safe self-contained carbon dioxide-hydrogen anaerobic system. Appl. Microbiol. 14:985-988.

6. Cleland, W. W. 1964. Dithiothreitol. A new protective reagent for SH groups. Biochemistry 3:480-482.

7. Ellner, P. D., P. A. Granato, and C. B. May. 1973. Recovery and identification of anaerobes: a system suitable for the routine clinical laboratory. Appl. Microbiol. 26:904-913.

8. Holdeman, L. V., E. P. Cato, and W. E. C. Moore (ed). 1977. Anaerobe laboratory manual, 4th ed. Anaerobe Laboratory, Virginia Polytechnic Institute and State University, Blacksburg.

9. Hungate, R. E.1969. A roll tube method for cultivation of strict anaerobes, p. 117-132. In J. R. Norris and D. W. Ribbons (ed.), Methods in microbiology, vol. 3B. Academic Press, Inc., London.

10. Mandel, M., and J. Marmur. 1968. Use of ultraviolet absorbance temperature profile for determining the guanine plus cytosine content of DNA. Methods Enzymol. 12B:195-206.

11. Marmur, J., and P. Doty. 1962. Determination of the base composition of deoxyribonucleic acid from its thermal denaturation point. J. Mol. Biol. 5:109-118.

12. Miles, A. A., and S. S. Misra. 1938. The estimation of the bacterial power of the blood. J. Hyg. 38:732-749.

13. Moore, W. B. 1968. Solidified media suitable for the cultivation of Clostridium novyi type B. J. Gen. Microbiol. 53:415-423.

14. Peckham, M. C. 1972. Ulcerative enteritis (quail disease), p. 242-250. In M. S. Hofstat et al. (ed.), Diseases of poultry, 8th ed. Iowa State University Press, Ames. 
15. Prévot, A. R. 1966. Manual for classification and determination of the anaerobic bacteria, 1st American ed. Translated by V. Fredette. Lea \& Febiger, Philadelphia.

16. Skerman, V. B. D., V. McGowan, and P. H. A. Sneath (ed.). 1980. Approved lists of bacterial names. Int. J. Syst. Bacteriol. 30:225-420.
17. Smith, L.DS. 1975. The pathogenic anaerobic bacteria. Charles C Thomas, Publisher, Springfield, Ill.

18. Smith, L.DS., and G. Hobbs. 1974. Genus III. Clostridium, p. 551-572. In R. E. Buchanan and N. E. Gibbons (ed.), Bergey's manual of determinative bacteriology, 8th ed. The Williams \& Williams Co., Baltimore. 